\title{
Perspective
}

\section{Chemokines and atherosclerosis: focus on the CX3CL1/CX3CR1 pathway}

\author{
Stavros APOSTOLAKIS, Demetrios SPANDIDOS* \\ Department of Clinical Virology, Faculty of Medicine, University of Crete, Heraklion, Crete, Greece
}

\begin{abstract}
Atherosclerosis is currently considered an inflammatory disease. Much attention has been focused on the potential role of inflammatory mediators as prognostic/diagnostic markers or therapeutic targets of atherosclerotic cardiovascular disease. CX3CL1 (or fractalkine) is a structurally and functionally unique chemokine with a well documented role in atherosclerosis. In its membrane bound form it promotes the firm adhesion of rolling leucocytes onto the vessel wall, while in its soluble form it serves as a potent chemoattractant for CX3CR1-expressing cells. Additionally, CX3CL1 exerts cytotoxic effects on the endothelium as well as anti-apoptotic and proliferative effects on vascular cells, affecting the context and stability of the atherosclerotic plaque. Studies on animal models have shown that the blockade of the CX3CL1/CX3CR1 pathway ameliorates the severity of atherosclerosis, while genetic epidemiology has confirmed that a genetically-defined less active CX3CL1/CX3CR1 pathway is associated with a reduced risk of atherosclerotic disease in humans. Although several studies support an important pathogenic role of CX3CL1/CX3CR1 in atherogenesis and plaque destabilization, this does not necessarily suggest that this pathway is a suitable therapeutic target or that CX3CL1 can serve as a prognostic/ diagnostic biomarker. Further studies on the CX3CL1/CX3CR1 chemokine pathway are clearly warranted to justify the clinical relevance of its role in atherosclerosis.
\end{abstract}

Keywords: atherosclerosis; chemokines; CX3CL1; CX3CR1; fractalkine

Acta Pharmacologica Sinica (2013) 34: 1251-1256; doi: 10.1038/aps.2013.92; published online 26 Aug 2013

\section{Introduction}

Despite advances in its diagnosis and treatment, vascular disease remains a leading cause of mortality worldwide ${ }^{[1,2]}$. Inflammation has been considered an important component of vascular disease ${ }^{[3]}$. The response-to-injury hypothesis proposed by Ross and Glomset suggests that the development of atherosclerotic lesions is the outcome of injury to the arterial endothelium that initiates the interaction of cellular populations of peripheral blood with cell components of the arterial wall $^{[4,5]}$. The progression of this inflammatory response is regulated by specific patterns of cytokine expression. The balance of pro- and anti-inflammatory cytokines is of great importance in the process of vascular inflammation.

The response to injury hypothesis remains an active field of investigation and multiple pro-inflammatory factors, chemoattractant cytokines and adhesion molecules have been proven important mediators in orchestrating this process ${ }^{[4-6]}$. Sadly, despite over three decades of research on the role of inflammation and atherosclerosis, no inflammatory mediators have

\footnotetext{
* To whom correspondence should be addressed.

E-mail spandidos@spandidos.gr

Received 2013-05-06 Accepted 2013-06-24
}

been applied in the diagnosis, prognosis or therapy of atherosclerotic vascular disease.

In the following review article we present an overview of the role of chemokines in atherosclerosis with particular focus on the CX3CR1/CX3CL1 (fractalkine) pathway. We further attempt a critical review of the available data in an effort to explain why promising in vitro data never become clinically applicable practices.

\section{Chemokines: structure and function}

Chemokines are a family of low molecular weight chemotactic cytokines (approximately 8-17 kDa) ${ }^{[7]}$. Their main function is to coordinate leukocyte trafficking and activation in health and disease $^{[7]}$. Chemokines share considerable homology and most importantly, a conserved tetracysteine motif. They are classified into four sub-families (C, CC, CXC, and CX3C) based on the number and structural arrangement of conserved cysteine residues within their amino-terminal polypeptide sequence ${ }^{[8]}$.

Chemokines signal through $G$ protein-coupled 7-transmembrane receptors to promote the directed migration of cells along a chemoattractant gradient. Following interaction with their specific chemokine ligands, chemokine receptors trigger a flux in intracellular calcium ions leading to dynamic assem- 
bly of the actin cytoskeleton and cellular movement ${ }^{[9]}$. There have been 19 distinct chemokine receptors identified in mammals.

Chemokine ligands also bind to decoy receptors - also known as interceptors (internalizing receptors) ${ }^{[10]}$. These receptors bind chemokine ligands with high affinity but do not educe signal transduction. There are three decoy receptors currently known: D6, Daffy antigen receptor for chemokines (DARC) and CCX-CKR. Chemokine receptor CCX-CKR is a scavenger of CCR7 ligand chemokines, while D6 is considered to be a chemokine scavenger for pro-inflammatory $\mathrm{CC}$ chemokines $^{[10]}$. DARC is a decoy receptor for both CC and CXC motif chemokines while no data exist on its affinity for CX3C and XC chemokines ${ }^{[11]}$.

\section{Chemokines and atherosclerosis}

Atherosclerotic vascular disease is characterized by complex chemokine signaling with different chemokine pathways involved in the different stages of disease ${ }^{[12]}$. In the early stages of atherogenesis, oxLDL induces the expression of CCL2 [or monocyte chemotactic protein-1 (MCP-1)] and CX3CL1 by vascular smooth muscle cells and endothelial cells ${ }^{[13]}$. CCL2 is secreted by endothelial cells and smooth muscle cells and promotes the transendothelial migration of CCR2-expressing monocytes ${ }^{[14]}$. CCL5 [or regulated on activation normal $\mathrm{T}$ cell expressed and secreted (RANTES)] through interaction with its receptor CCR1, is also considered an early pathway leading to accumulation of rolling monocytes to stimulated endothelial cells ${ }^{[13,14]}$. CXC chemokines induced by interferon gamma, such as CXCL9 [or monokine induced by gamma interferon (MIG)], CXCL10 [or interferon gamma-induced protein 10 (IP-10)] and CXCL11 [or interferon-inducible T-cell alpha chemoattractant (I-TAC)] expressed predominantly by endothelial cells interact with CXCR3-positive T cells, inducing their accumulation and migration to the inflamed vascular wall ${ }^{[13,14]}$. The recruitment of neutrophils and vascular progenitor cells in atherosclerotic lesions is controlled by CXCR2 and CXCR4, and their ligand, CXCL8 (or interleukin 8). CXCL8 is highly expressed by lesion macrophages as well as by endothelial cells and smooth muscle cells. CXCL8 also promotes angiogenesis in advanced stages of plague development ${ }^{[15]}$. It is obvious that during the course of atherosclerosis, chemokines form a complex network. Different chemokines promote different pathways. Moreover, the interaction of the same chemokine ligand with different receptors results in a different outcome. In such a complex system the consequences of a single pathway blockade cannot be predicted ${ }^{[16]}$.

\section{CX3CL1 (fractalkine): unique structure and function}

CX3CL1 (or fractalkine) is an atypical chemokine with a documented role in the development of numerous inflammatory diseases, including atherosclerosis. It represents the only known member of the CX3C family and was first characterized by Bazan et al in 1997 ${ }^{[17]}$. It is a unique chemotactic factor existing in both the membrane-bound and soluble form ${ }^{[17]}$. The human CX3CL1 molecule consists of 373 amino acids and it is functionally divided into 4 domains: an extracellular domain of 76 amino acids connected to an extended 241 amino acid mucin-like stalk, followed by transmembrane and intracellular domains of 21 and 35 amino acids, respectively. CX3CL1 is synthesized as a membrane-bound molecule with the chemokine domain presented on a mucin-like stalk which mediates the direct capture of circulating leukocytes ${ }^{[17]}$. Cleavage at the base of this stalk by a protease, namely the tumor necrosis alpha-converting enzyme, generates a soluble chemokine, which functions as a potent chemoattractant of target cells ${ }^{[17,18]}$. Its unique structure results in unique function, as CX3CL1 is the only known chemokine able to act both as a chemoattractant and as an efficient adhesion molecule through a non-integrin dependent mechanism. CX3CL1 signals through a single G-protein-linked receptor: CX3CR1 ${ }^{[18,19]}$. Unlike most chemokine ligands that demonstrate affinity for multiple receptors, CX3CL1 interacts with a single receptor. CX3CR1 is expressed on monocytes, natural killer cells, T cells, and smooth muscle cells, where it mediates migration, adhesion and proliferation ${ }^{[20]}$. Promotion of cell survival is considered another key function of CX3CL1 operating under both homeostatic and inflammatory conditions ${ }^{[20]}$.

\section{CX3CL1 and atherosclerosis: data from ex vivo studies and animal models}

CX3CL1 has been considered an important mediator in all stages of atherogenesis, including the recruitment of monocytes in early atherosclerotic lesions, as well as the promotion of vascular smooth muscle cell migration and neo-angiogenesis in more advanced plaques (Figure 1).

Early immunochemical studies have confirmed CX3CL1 expression in monocytes/macrophages, endothelial and smooth muscle cells within human atherosclerotic coronary

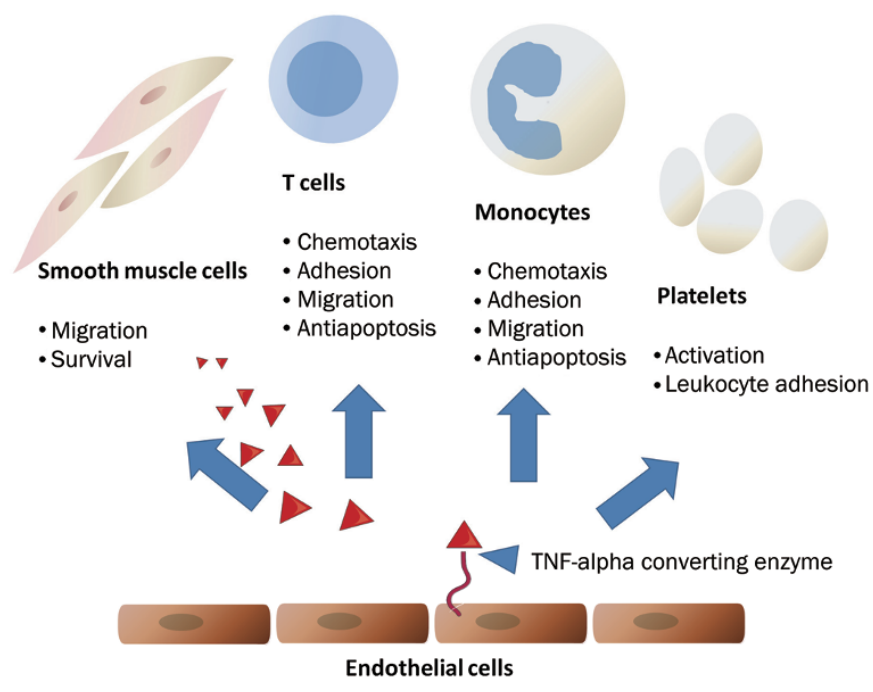

Figure 1. CX3CL1 mediated pathways in atherosclerosis. CX3CL1 is synthesized as a membrane-bound molecule with the chemokine domain presented on a mucin-like stalk. Cleavage at the base of this stalk tumor necrosis alpha-converting enzyme, generates a soluble chemokine, which functions as a potent chemoattractant of target cells. 
arteries $^{[21]}$. Lucas et al detected the expression of CX3CR1 in smooth muscle cells in the neointima of human atherosclerotic plaques. The authors further demonstrated that primary cultured human coronary artery smooth muscle cells migrate toward CX3CL1, suggesting that CX3CR1 promotes the migration of smooth muscle cells in more advanced stages of atherosclerosis $^{[22]}$. Using immunohistochemistry and quantitative PCR, Stolla et al confirmed that CX3CL1 is expressed in all stages of atherogenesis, in human carotid artery plaques ${ }^{[23]}$.

In a different setting, Shah et al demonstrated that CX3CL1 is expressed in adipocytes and stromal vascular cells in a human endotoxemia model. The authors concluded that CX3CL1/ CX3CR1 is a novel inflammatory adipose chemokine pathway that modulates monocyte adhesion to adipocytes and is associated with obesity, insulin resistance and type 2 diabetes ${ }^{[24]}$.

Two independent studies have demonstrated a significant decrease in atherosclerotic lesion size in the aorta of mice lacking the CX3CL1/CX3CR1 signaling mechanism ${ }^{[25,26]}$, while more recently, Saederup et al demonstrated that the deletion of CX3CL1 in CCR2-deficient mice dramatically reduced the accumulation of macrophages in the arterial wall and the subsequent development of atherosclerosis ${ }^{[27]}$. The latter study concluded that successful therapeutic strategies may need to target multiple chemokines and/or chemokine receptors $^{[27]}$. In a similar setting, Combadière et al demonstrated that the combined inhibition of CCL2, CX3CR1, and CCR5 in hypercholesterolemic, atherosclerosis-susceptible apolipoprotein E-deficient mice leads to the abrogation of bone marrow monocytosis and to additive reduction in circulating monocytes despite persistent hypercholesterolemia. These effects were associated with a marked and additive $90 \%$ reduction in atherosclerosis $^{[28]}$. More recently, Liu et al, using both RT-PCR and immunohistochemical analysis, showed that CX3CL1 expression was higher in high-fat- vs normal diet-fed ApoE gene knockout mice and was significantly decreased by aspirin treatment ${ }^{[29]}$.

Recent studies have also implicated CX3CL1 in platelet stimulation and activation. Platelets have been shown to express CX3CR1 and exposure to CX3CL1 has been shown to promote platelet activation. Schäfer et al demonstrated that platelets from rats, pre-incubated with CX3CL1, had increased P-selectin surface expression. Also, pre-incubation with CX3CL1 enhanced platelet adhesion to collagen and fibrinogen $^{[30]}$. In the field of platelet endothelial interaction, Schulz et al demonstrated that CX3CL1 expressed by inflamed endothelial cells triggers P-selectin exposure on adherent platelets, which thereby initiates the local accumulation of leukocytes under arterial shear, an essential step in the development of atherosclerotic lesions ${ }^{[31]}$.

CX3CL1 has also been demonstrated to exert cytotoxic effects on the vascular endothelium, as well as anti-apoptotic and proliferative effects on vascular cells, affecting the synthesis and stability of the atherosclerotic plaque ${ }^{[32]}$.

The role of CX3CL1/CX3CR1 in atherosclerosis is summarized in Figure 2.

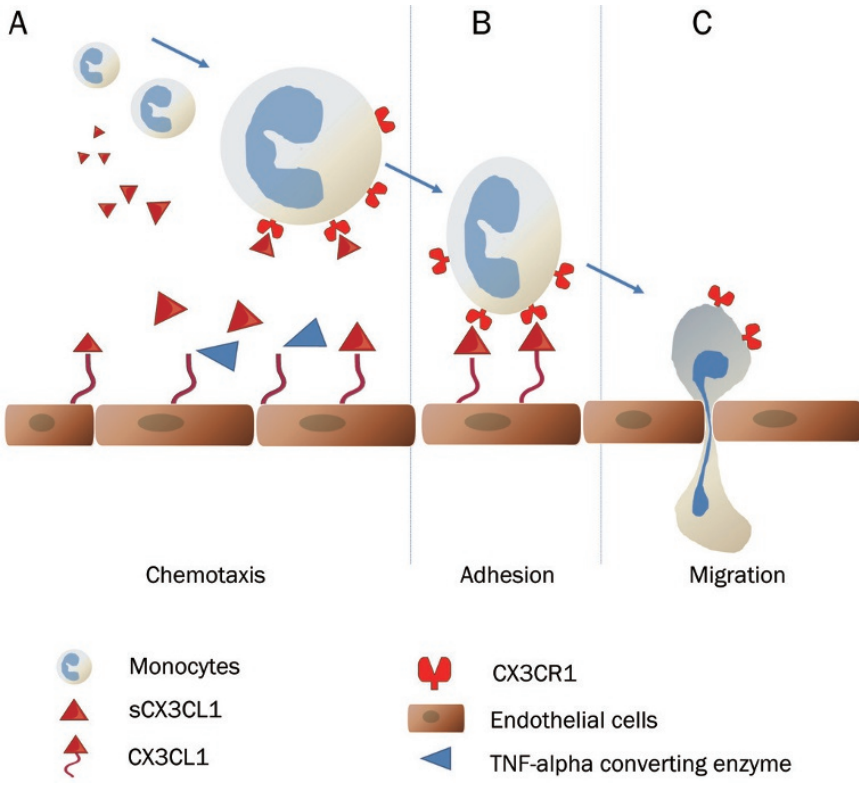

Figure 2. The dual-role of CX3CL1 in atherosclerosis: In its membrane bound form, it promotes the firm adhesion of rolling leucocytes onto the vessel wall (B) promoting sub-endothelial migration (C). In its soluble form, following cleavage by the TNF-alpha converting enzyme, it serves as a potent chemoattractant for CX3CR1-expressing cells (A).

\section{CX3CL1 and atherosclerosis: clinical research}

Commonly used clinical risk factors, individually or incorporated in risk algorithms, fail to identify all individuals at risk of cardiovascular disease. This notion has inspired biomarker research in the field of cardiovascular disease prevention for more than 3 decades. An ideal biomarker should provide diagnostic information in patients at risk for cardiovascular disease and/or prognostic information on patients with established cardiovascular disease and/or serve as predictors of efficacy for therapeutic interventions.

Serum levels of CX3CL1 have been assessed as a potential diagnostic and prognostic biomarker. Stolla et al assessed soluble CX3CL1 serum levels in 137 patients with atherosclerotic carotid artery disease. The authors observed significantly elevated levels of soluble CX3CL1 in patients with carotid artery stenosis near occlusion (90\%-99\% stenosis) but similar levels among patients with moderate (50\%-70\% stenosis) and advanced (70\%-90\% stenosis) carotid artery disease ${ }^{[23]}$. Yadav et al assessed 62 healthy controls and 128 patients with stage III-V chronic kidney disease $(\mathrm{CKD})^{[33,34]}$. The authors observed increased levels of soluble CX3CL1 in CKD subjects and a significant correlation between common carotid artery intima-media thickness and soluble CX3CL1 levels. In a case-control study involving 281 type 2 diabetics and 274 age and gender-matched controls, Shah et al observed increased plasma CX3CL1 levels in patients with type 2 diabetes vs non diabetics ${ }^{[24]}$. Nevertheless, in a large cohort of angiographically verified coronary artery disease (CAD) patients, Njerve et al detected no differences in circulating levels of CX3CL1 between patients with or without type II diabetes mellitus, 
or between patients with or without metabolic syndrome ${ }^{[35]}$. Maegdefessel et al compared serum CX3CL1 concentrations among 46 patients with coronary heart disease, 47 insulindependent diabetic patients following rehabilitation and 50 control subjects. The authors demonstrated that following rehabilitation serum, CX3CL1 levels in coronary heart disease patients were similar to those in control subjects, whereas CX3CL1 levels were lower in insulin-dependent diabetic patients ${ }^{[36]}$. Li et al assessed the concentrations and mRNA expression levels of CCL2, CCL5, and CX3CL1 in 60 patients with acute myocardial infarction, 60 patients with unstable angina pectoris, 60 patients with stable angina pectoris and 40 patients without coronary heart disease. The authors observed significantly higher chemokine levels in unstable coronary artery disease patients compared to stable coronary artery disease patients ${ }^{[37]}$. Similarly, Damås et al assessed plasma levels of CX3CL1 and CX3CR1 expression in peripheral blood mononuclear cells of various coronary artery disease populations and concluded that the plasma levels of CX3CL1 are greatly increased in coronary artery disease, particularly in unstable angina with parallel increase of CX3CR1 expression in peripheral blood mononuclear cells. The authors further demonstrated that statin therapy for 6 months reduced the expression of CX3CL1 and CX3CR1 $1^{[38]}$. Our group, using flow cytometry, similarly observed a higher rate of CX3CR1-positive peripheral blood mononuclear cells in patients with coronary artery disease as compared to the controls with a difference that was enhanced beyond the limit of statistical significance when CX3CR1 expression was evaluated in the monocyte gate ${ }^{[39]}$.

In a different cardiovascular disease setting, Richter et al assessed CX3CL1 plasma levels in 349 patients with advanced systolic heart failure. The authors observed over a median follow-up of 4.9 years that CX3CL1 was a significant predictor of all-cause mortality with a hazard ratio of 2.78 for the third compared to the first tertile ${ }^{[40]}$. The same group subsequently demonstrated that a multi-biomarker score including plasma CX3CL1 levels had strong discriminatory power for 5-year mortality (area under the Receiver Operating Characteristic curve 0.81 ) in patients with systolic heart failure ${ }^{[41]}$.

In support of this evidence, several population-based genetic studies conducted in different population samples evaluated the effect of genetic variations of the CX3CL1 receptor on susceptibility to coronary artery disease. Two non-synonymous polymorphisms have been identified in the CX3CR1 gene ${ }^{[42-44]}$. They result in a valine to isoleucine substitution at position 249 (CX3CR1-V249I), and threonine to methionine substitution at position 280 (CX3CR1-T280M) in the final polypeptide sequence of the final CX3CR1 protein ${ }^{[41]}$. These changes are located in the sixth and seventh transmembrane domains, respectively ${ }^{[42]}$. Several independent studies conducted in different settings of atherosclerotic cardiovascular disease have revealed an atheroprotective effect of these common single nucleotide polymorphisms of the $\mathrm{CX} 3 \mathrm{C}$ receptor gene attributed to the reduced activity of the CX3CL1/CX3CR1 pathway ${ }^{[42-44]}$. Accordingly, a meta-analysis involving 2000 coronary artery disease patients and 2841 subjects without evidence of cardiovascular disease, confirmed a strong association between the $280 \mathrm{M}$ allele and reduced risk for coronary artery disease in the heterozygous state ${ }^{[45]}$.

\section{From bench to bedside: the future of CX3CL1}

Since the establishment of the inflammatory basis of atherosclerosis, several pro- or anti-inflammatory agents have been examined as potential mediators of the biochemical pathways of atherogenesis. Following the discovery of the first chemokine in 1987, almost every one of the currently 46 known family members has been implicated, in aspects of cardiovascular disease in general and atherosclerotic vascular disease in particular ${ }^{[46]}$.

The structural and functional uniqueness of CX3CL1, demonstrated by multiple studies under different settings, have clearly established a key role for the CX3CL1/CX3CR1 pathway in atherogenesis. Nevertheless, how the information that has been derived from in vitro studies and animal models can be applied in clinical settings remains to be determined.

The management of atherosclerotic cardiovascular disease consists of primary prevention, secondary prevention, and treatment of acute complications of atherosclerosis. Primary prevention requires overall cardiovascular risk estimation and risk factor modification. Commonly used risk algorithms, are quite effective but fail to identify all individuals that are potentially affected by cardiavscular disease. Novel cardiovascular biomarkers that identify these missed individuals would greatly improve current primary prevention practices. In cardiovascular risk estimation, several reports have indicated that increased serum levels of CX3CL1 correlate with an increased risk of cardiovascular disease per se, an adverse cardiovascular risk profile or acute cardiovascular events. However, these data are primarily the results of single-centre small-scale observational studies. There are no data available derived from large-scale prospective clinical trials illustrating the additive value of CX3CL1 on traditional risk factors as prognostic markers of short- or long-term outcome after acute cardiovascular events or in a primary prevention setting. There are also methodological issues in the assessment of chemokines as biomarkers. Do plasma levels or serum levels of chemokines best reflect the underlying inflammatory process? Recently, Schnabel $e$ t al observed that serum values of CCL2 are almost twice those in plasma. The authors demonstrated that this phenomenon is likely due to the strong binding of CCL2 in erythrocyte DARC receptors and the release of CCL2 by activation of anticoagulation $^{[47]}$. Erythrocyte DARC represents a large reservoir of chemokines and perhaps DARC-bound chemokines would be better biomarkers of atherosclerotic cardiovascular disease. In support of the latter theory, Tziakas et al demonstrated an association between erythrocyte-bound but not plasma CXCL8 and the clinical presentation of coronary artery disease, suggesting that erythrocyte-bound CXCL8 might be a better marker of unstable coronary artery disease ${ }^{[48]}$. Thus, there are a lot of obscure aspects in the regulation of plasma chemokines and it is likely that CX3CL1 plasma concentrations might also be regulated by a yet unknown scavenging system. 
Another issue is whether additional markers for atherosclerosis are required in either a primary or a secondary setting. Inflammatory markers have to demonstrate high specificity and sensitivity and increase predictive value positively or negatively, particularly if we take into account cost-efficiency parameters and the fact that most traditional risk factors are highly modifiable.

As regards the therapeutic potential of the CX3CL1/ CX3CR1 pathway, only limited data are available. Data from animal models are encouraging, demonstrating the suppression of atherosclerosis when the CX3CR1/CX3CL1 pathway is blocked. From the efficacy point of view, one key question is whether the long-term inhibition of a single chemokine/receptor would be an effective therapeutic strategy. The combined inhibition of CCL2 and CX3CL1 may be required to overcome redundancy in the chemokine network. Indeed, blockade of multiple chemokine-mediated pathways at the level of the receptor has been proven far more effective than the blockade of single pathways ${ }^{[27,28]}$. From the safety point of view, chemokine inhibition as any immune-modulating therapeutic approach has several drawbacks that need to be considered. The resulting chronic monocyte suppression could have significant undesirable effects. Although targeting CX3CL1 in early atherosclerosis might reduce monocyte accumulation and survival in fatty streak lesions, macrophage survival may be crucial in late disease stages to ensure the clearance of necrotic and potentially thrombogenic material. Additionally, atherosclerosis is a chronic condition; thus, long-term therapy must be applied. This raises tolerability and safety issues for a potential therapeutic agent that blocks a non-specific chemotactic pathway. Moreover, CX3CL1 is an important mediator of several aspects of immune response. Therefore, the complete blockade of CX3CL1 signaling pathways may not be desirable. Thus, techniques need to be applied to ensure that CX3CL1 signal blocking takes place in specific areas and tissues. An appealing area of investigation for immunesuppressive therapies is restenosis after percutaneous coronary intervention(PCI), in which CX3CL1 signalling blockers may be tested as potential agents for stent eluting. This latter approach ensures better targeting and probably less side effects than systemic treatment.

Nonetheless, it is necessary to take into account that all therapeutic approaches for cardiovascular disease have been evaluated in large-scale clinical trials with specific "hard" endpoints. Therefore, despite its promising in vivo and in vitro data, any CX3CL1 modulating therapy must be proven to exhibit the ability to reduce cardiovascular morbidity and mortality. To the best of our knowledge, no large-scale clinical trials are currently evaluating the effectiveness of CX3CL1-as a prognostic marker or therapeutic target. It will therefore be a long wait before CX3CL1-based therapeutic approaches find their way into clinical practice.

In conclusion, there is sufficient evidence in the scientific literature to support an important role of CX3CL1/CX3CR1 in atherosclerosis. There is as yet no convincing evidence to support potential clinical use of CX3CL1 as a prognostic marker, diagnostic marker or therapeutic target in atherosclerotic vascular disease.

\section{References}

1 Mirzaei M, Truswell AS, Taylor R, Leeder SR. Coronary heart disease epidemics: not all the same. Heart 2009; 95: 740-6.

2 European Heart Network. European cardiovascular disease statistics. 2008 edition.

3 Ross R. Atherosclerosis - an inflammatory disease. N Engl J Med 1999; 340: 115-26.

4 Ross R, Glomset JA. The pathogenesis of atherosclerosis (second of two parts). N Engl J Med 1976; 295: 420-5.

5 Ross R, Glomset JA. The pathogenesis of atherosclerosis (first of two parts). N Engl J Med 1976; 295: 369-77.

6 Weber C. Chemokines in atherosclerosis, thrombosis, and vascular biology. Arterioscler Thromb Vasc Biol 2008; 28: 1896.

7 Luster AD. Chemokines - chemotactic cytokines that mediate inflammation. N Engl J Med 1998; 338: 436-45.

8 IUIS/WHO subcommittee on chemokine nomenclature. Chemokine/ chemokine receptor nomenclature. Cytokine 2003; 21: 48-9.

9 Jin T, Xu X, Hereld D. Chemotaxis, chemokine receptors and human disease. Cytokine 2008; 44: 1-8.

10 Comerford I, Nibbs RJ. Post-translational control of chemokines: a role for decoy receptors? Immunol Lett 2005; 96: 163-74.

11 Apostolakis S, Chalikias GK, Tziakas DN, Konstantinides S. Erythrocyte Duffy antigen receptor for chemokines (DARC): diagnostic and therapeutic implications in atherosclerotic cardiovascular disease. Acta Pharmacol Sin 2011; 32: 417-24.

12 Cushing SD, Berliner JA, Valente AJ, Territo MC, Navab M, Parhami F, et al. Minimally modified low density lipoprotein induces monocyte chemotactic protein 1 in human endothelial cells and smooth muscle cells. Proc Natl Acad Sci U S A 1990; 87: 5134-8.

13 Weber C, Schober A, Zernecke A. Chemokines: key regulators of mononuclear cell recruitment in atherosclerotic vascular disease. Arterioscler Thromb Vasc Biol 2004; 24: 1997-2008.

14 Barlic J, Murphy PM. Chemokine regulation of atherosclerosis. J Leukoc Biol 2007; 82: 226-36.

15 Apostolakis S, Vogiatzi K, Amanatidou V, Spandidos DA. Interleukin 8 and cardiovascular disease. Cardiovasc Res 2009; 84: 353-60.

16 Apostolakis S, Amanatidou V, Spandidos DA. Therapeutic implications of chemokine-mediated pathways in atherosclerosis: realistic perspectives and utopias. Acta Pharmacol Sin 2010; 31: 1103-10.

17 Bazan JF, Bacon KB, Hardiman GJ. A new class of membrane bound chemokine with a CX3C motif. Nature 1997; 385: 640-4.

18 Haskell CA, Cleary MD, Charo IF. Unique role of the chemokine domain of fractalkine in cell capture. Kinetics of receptor dissociation correlate with cell adhesion. J Biol Chem 2000; 275: 34183-9.

19 Imai T, Hieshima K, Haskell C, Baba M, Nagira M, Nishimura M, et al. Identification and molecular characterization of fractalkine receptor CX3CR1, which mediates both leukocyte migration and adhesion. Cell 1997; 91: 521-30.

20 White GE, Greaves DR. Fractalkine: a survivor's guide: chemokines as antiapoptotic mediators. Arterioscler Thromb Vasc Biol 2012; 32: 589-94.

21 Wong BW, Wong D, McManus BM. Characterization of fractalkine (CX3CL1) and CX3CR1 in human coronary arteries with native atherosclerosis, diabetes mellitus, and transplant vascular disease. Cardiovasc Pathol 2002; 11: 332-8.

22 Lucas AD, Bursill C, Guzik TJ, Sadowski J, Channon KM, Greaves DR. Smooth muscle cells in human atherosclerotic plaques express the fractalkine receptor CX3CR1 and undergo chemotaxis to the CX3C 
chemokine fractalkine (CX3CL1). Circulation 2003; 108: 2498-504.

23 Stolla M, Pelisek J, von Brühl ML, Schäfer A, Barocke V, Heider P, et al. Fractalkine is expressed in early and advanced atherosclerotic lesions and supports monocyte recruitment via CX3CR1. PLoS One 2012; 7: e43572.

24 Shah R, Hinkle CC, Ferguson JF, Mehta NN, Li M, Qu L, et al. Fractalkine is a novel human adipochemokine associated with type 2 diabetes. Diabetes 2011; 60: 1512-8.

25 Combadiere C, Potteaux S, Gao JL, Esposito B, Casanova S, Lee EJ, et al. Decreased atherosclerotic lesion formation in CX3CR1/apolipoprotein E double knockout mice. Circulation 2003; 107: 1009-16.

26 Lesnik P, Haskell CA, Charo IF. Decreased atherosclerosis in $\mathrm{CX} 3 \mathrm{CR}^{-1-}$ mice reveals a role for fractalkine in atherogenesis. J Clin Invest 2003; 111: 333-40.

27 Saederup N, Chan L, Lira SA, Charo IF. Fractalkine deficiency markedly reduces macrophage accumulation and atherosclerotic lesion formation in $\mathrm{CCR}^{-/-}$mice: evidence for independent chemokine functions in atherogenesis. Circulation 2008; 117: 1642-8.

28 Combadière C, Potteaux S, Rodero M, Simon T, Pezard A, Esposito B, et al. Combined inhibition of CCL2, CX3CR1, and CCR5 abrogates Ly6C(hi) and Ly6C(Io) monocytosis and almost abolishes atherosclerosis in hypercholesterolemic mice. Circulation 2008; 117: 1649-57.

29 Liu H, Jiang D, Zhang S, Ou B. Aspirin inhibits fractalkine expression in atherosclerotic plaques and reduces atherosclerosis in $A p o E$ gene knockout mice. Cardiovasc Drugs Ther 2010; 24: 17-24.

30 Schäfer A, Schulz C, Eigenthaler M, Fraccarollo D, Kobsar A, Gawaz $\mathrm{M}$, et al. Novel role of the membrane-bound chemokine fractalkine in platelet activation and adhesion. Blood 2004; 103: 407-12.

31 Schulz C, Schäfer A, Stolla M, Kerstan S, Lorenz M, von Brühl ML, et al. Chemokine fractalkine mediates leukocyte recruitment to inflammatory endothelial cells in flowing whole blood: a critical role for P-selectin expressed on activated platelets. Circulation 2007; 116: 764-73.

32 Liu H, Jiang D. Fractalkine/CX3CR1 and atherosclerosis. Clin Chim Acta 2011; 412: 1180-6.

33 Yadav AK, Lal A, Jha V. Association of circulating fractalkine (CX3CL1) and CX3CR1(+)CD4(+) T cells with common carotid artery intimamedia thickness in patients with chronic kidney disease. J Atheroscler Thromb 2011; 18: 958-65.

34 Yadav AK, Sharma V, Jha V. Association between serum neopterin and inflammatory activation in chronic kidney disease. Mediators Inflamm 2012; 2012:476979.

35 Njerve IU, Pettersen Å̊, Opstad TB, Arnesen H, Seljeflot I. Fractalkine and its receptor (CX3CR1) in patients with stable coronary artery disease and diabetes mellitus. Metab Syndr Relat Disord 2012; 10 : 400-6.

36 Maegdefessel L, Schlitt A, Pippig S, Schwaab B, Fingscheidt K, Raaz $\mathrm{U}$, et al. Patients with insulin-dependent diabetes or coronary heart disease following rehabilitation express serum fractalkine levels similar to those in healthy control subjects. Vasc Health Risk Manag 2009;5: 849-57.

37 Li J, Guo Y, Luan X, Qi T, Li D, Chen Y, et al. Independent roles of monocyte chemoattractant protein-1, regulated on activation, normal T-cell expressed and secreted and fractalkine in the vulnerability of coronary atherosclerotic plaques. Circ J 2012; 76: 2167-73.

38 Damås JK, Boullier A, Waehre T, Smith C, Sandberg WJ, Green S, et al. Expression of fractalkine (CX3CL1) and its receptor, CX3CR1, is elevated in coronary artery disease and is reduced during statin therapy. Arterioscler Thromb Vasc Biol 2005; 25: 2567-72.

39 Apostolakis S, Krambovitis E, Vlata Z, Kochiadakis GE, Baritaki S, Spandidos DA. CX3CR1 receptor is up-regulated in monocytes of coronary artery diseased patients: impact of pre-inflammatory stimuli and renin-angiotensin system modulators. Thromb Res 2007; 121: 387-95.

40 Richter B, Koller L, Hohensinner PJ, Rychli K, Zorn G, Goliasch G, et al. Fractalkine is an independent predictor of mortality in patients with advanced heart failure. Thromb Haemost 2012; 108: 1220-7.

41 Richter B, Koller L, Hohensinner PJ, Zorn G, Brekalo M, Berger R, et al. A multi-biomarker risk score improves prediction of long-term mortality in patients with advanced heart failure. Int J Cardiol 2012; doi: 10.1016/j.ijcard.2012.11.052.

42 Moatti D, Faure S, Fumeron F, Amara Mel-W, Seknadji P, McDermott $\mathrm{DH}$, et al. Polymorphism in the fractalkine receptor CX3CR1 as a genetic risk factor for coronary artery disease. Blood 2001; 97 : 1925-8.

43 McDermott DH, Halcox JP, Schenke WH, Waclawiw MA, Merrell MN, Epstein N, et al. Association between polymorphism in the chemokine receptor CX3CR1 and coronary vascular endothelial dysfunction and atherosclerosis. Circ Res 2001; 89: 401-7.

44 Apostolakis S, Amanatidou V, Papadakis EG, Spandidos DA. Genetic diversity of CX3CR1 gene and coronary artery disease: new insights through a meta-analysis. Atherosclerosis 2009; 207: 8-15.

45 Apostolakis S, Baritaki S, Kochiadakis GE, Igoumenidis NE, Panutsopulos D, Spandidos DA. Effects of polymorphisms in chemokine ligands and receptors on susceptibility to coronary artery disease. Thromb Res 2007; 119: 63-71.

46 Yoshimura T, Matsushima K, Oppenheim JJ, Leonard EJ. Neutrophil chemotactic factor produced by lipopolysaccharide (LPS)-stimulated human blood mononuclear leukocytes: partial characterization and separation from interleukin 1 (IL 1). J Immunol 1987; 139: 788-93.

47 Schnabel RB, Baumert J, Barbalic M, Dupuis J, Ellinor PT, Durda P, et al. Duffy antigen receptor for chemokines (Darc) polymorphism regulates circulating concentrations of monocyte chemoattractant protein-1 and other inflammatory mediators. Blood 2010; 115: 5289-99.

48 Tziakas DN, Chalikias GK, Tentes IK, Stakos D, Chatzikyriakou SV, Mitrousi $\mathrm{K}$, et al. Interleukin-8 is increased in the membrane of circulating erythrocytes in patients with acute coronary syndrome. Eur Heart J 2008; 29: 2713-22. 